\title{
Cost and Impact on Patient Length of Stay of Rapid Molecular Testing for Clostridium difficile
}

\author{
Bernadette Sewell • Eugene Rees • Ian Thomas • \\ Chin Lye Ch'ng $\cdot$ Mike Isaac $\cdot$ Nidhika Berry
}

To view enhanced content go to www.infectiousdiseases-open.com

Received: July 7, 2014 / Published online: September 3, 2014

(c) The Author(s) 2014. This article is published with open access at Springerlink.com

\section{ABSTRACT}

Introduction: A study was performed to assess the cost of a rapid molecular assay (PCR) for diagnosis of Clostridium difficile infection (CDI) and the impact of its routine use on patient length of stay (LOS) in comparison with cell culture cytotoxin neutralization assay (CCNA).

Methods: From March 2011 to September 2011, Xpert $^{\circledR}$ C. difficile (Cepheid, Sunnyvale, CA, USA) PCR was used on patients with suspicion of CDI in two acute care hospitals in Abertawe Bro Morgannwg University Health Board, Swansea, Wales, UK. Test results were used for patient management. LOS and time to

Electronic supplementary material The online version of this article (doi:10.1007/s40121-014-0034-x) contains supplementary material, which is available to authorized users.

B. Sewell $(\bowtie)$

Swansea Centre for Health Economics, College of Human and Health Sciences, Swansea University, Singleton Park, Swansea, UK

e-mail: b.diethart@swansea.ac.uk

E. Rees · I. Thomas · M. Isaac · N. Berry

Public Health Wales, Microbiology ABMUHB,

Singleton Hospital, Swansea, UK

C. L. Ch'ng

Gastroenterology Department, Singleton Hospital, Swansea, UK reportable result were compared for negative and positive prospective patients tested by PCR and historic control patients tested by CCNA during March 2010 to September 2010. Tests were priced using micro-costing and a cost comparison analysis was undertaken.

Results: In total, 506 patients were included. Time to reportable result for PCR samples was $1.53 \mathrm{~h}$ compared to $46.54 \mathrm{~h}$ for CCNA negatives and $22.45 \mathrm{~h}$ for CCNA positives. Patients tested by CCNA stayed 4.88 days longer in hospital compared to PCR patients if they tested positive and 7.03 days if tests were negative. The mean reduction in LOS observed in our study has the potential to generate cost savings of up to $£ 2,292.62$ for every patient with suspected $\mathrm{CDI}$, if samples were to be tested routinely with PCR instead of CCNA.

Conclusion: A rapid molecular test for C. difficile in an acute hospital setting produced quick results that led to a decrease in LOS compared to historic CCNA control patients. This could result in considerable savings through reduced excess inpatient days.

Keywords: Cell culture cytotoxin neutralization assay; Clostridium difficile; 
Cost comparison; Length of hospital stay; Polymerase chain reaction (PCR); Rapid molecular testing; Xpert PCR

\section{INTRODUCTION}

Clostridium difficile is a fastidious anaerobe that causes nosocomial antibiotic-associated colitis, ranging from mild to severe disease, including pseudo-membranous colitis and toxic megacolon with a potentially fatal outcome [1]. Even though the pathogenesis, diagnosis and prevention of $C$. difficile infection (CDI) have received particular attention in recent years, CDI still remains a leading cause of healthcare-associated diarrhea with a profound clinical as well as economic impact [2]. Estimates of the financial burden of CDI have been estimated to be between $\$ 2,454$ and $\$ 16,464$ for every healthcare-acquired CDI case in the US [3-5], $£ 4,107$ in the UK [6], and $€ 7,147$ in Germany [7]. The length of hospital stay (LOS) has been identified as the main cost driver in most economic studies of CDI [3, 4, 6], with patients suffering from nosocomial CDI staying on average between 3 and 26 days longer than patients without CDI [6-9]. Furthermore, an increase in LOS due to more severe disease was observed in recent studies [10]. It has been suggested that this may be partly attributable to long turnaround times of assays and algorithms used to detect the presence of $C$. difficile in stool samples [11]. The cell culture cytotoxin neutralization assay (CCNA) and also toxigenic culture are historically considered to be the gold standard assays for $C$. difficile detection $[12,13]$. However, CCNA usually takes around $48 \mathrm{~h}$ until results can be reported and it requires the ability to perform cell culture [12]. Recent developments in testing for CDI include commercial and in-house polymerase chain reaction (PCR), as well as glutamate dehydrogenase (GDH) enzyme-based tests. GDH assays require $4-6 \mathrm{~h}$ from receipt until reportable results are available. GDH detects toxigenic as well as non-toxigenic strains and while it has been recommended as a screening tool in combination with other confirmative tests for GDH-positive samples [13, 14], its sensitivity was reported to be less than optimal $[6,15]$. Although the performance of PCR assays was found to exceed the clinical performance of GDH-based individual tests and algorithms [15], in-house molecular assays require technical expertise and additional capital expenses. Acquisition cost of commercially available kitbased PCR assays are considered to be higher compared to GDH or CCNA [16], but it has been proposed that increased sensitivity of PCR could ultimately lead to cost savings due to more accurate diagnosis and reduced repeat testing [15]. Faster turnaround time from testing to reporting may result in shorter LOS and decreased risk of transmission. The impact of molecular methods for $C$. difficile detection on duration of hospital stay compared to other assays and potential cost savings due to shorter hospital stays or fewer repeat samples has yet to be determined.

In a prospective trial carried out in two acute care hospitals in Swansea, UK, the clinical utility of the real-time PCR test Xpert ${ }^{\circledR} C$. difficile (Cepheid, Sunnyvale, CA, USA) was assessed in comparison to CCNA. Xpert C. difficile was found to be easy to use, rapid ( $<1 \mathrm{~h}$ run time), clinically useful, sensitive, and reliable in CDI diagnosis [17].

The aim of this cost comparison study was to assess the cost of $C$. difficile PCR and its impact on LOS for patients with suspicion of CDI in an acute hospital site compared to CCNA as the conventional diagnostic reference method. 


\section{METHODS}

The cost comparison study was conducted in parallel with a clinical study run at two acute hospital sites within the Abertawe Bro Morgannwg University Health Board (ABMUHB) between March 2011 and September 2011. This study investigated the sensitivity and specificity of PCR, CCNA, GDH, and a two-step GDH/toxin enzyme immunoassay (EIA) algorithm with clinical diagnosis as the Ref. [17]. Routinely collected stool samples of patients with suspected CDI were tested for the presence of $C$. difficile using real-time PCR (GeneXpert ${ }^{\circledR}$ testing platform; Cepheid, Sunnyvale, CA, USA) and two-step GDH/toxin EIA enzyme immunoassay (Premier $^{\circledR}$ on a Dynex DS2 ${ }^{\circledR}$ testing platform; Dynex Technologies, Chantilly, VA, USA), in addition to routine CCNA. The clinical study [17] included patients $>15$ years with 2 or more unformed stools (Bristol stool chart 5-7) within a 24-h period who had been admitted to hospital no shorter than 3 days before sample collection to exclude community origin of disease. PCR and CCNA results were reported to the respective wards through the Laboratory Information System as soon as they became available. Once positives were identified, patients were managed according to standard clinical protocols for treatment of CDI [17]. All positive PCR and/or CCNA results were additionally phoned to the wards or infection control nurses. Patients were immediately isolated in a side room, if available, prior to microbiological diagnosis, as per ABMUHB policy.

The first 150 PCR-positive and 150 PCRnegative patients of the clinical study were planned to be included in the cost comparison study. Separate from the ongoing clinical study, as a control, patients with positive and negative
PCR samples were age and gender matched to patients with positive or negative CCNA results from the same calendar month in the previous year. This led to the formation of four patient groups comprising PCR-positive, PCR-negative, CCNA-positive, and CCNA-negative patients. Due to the fact that the clinical study focused on diagnostic accuracy of various tests for $C$. difficile detection in stool samples, GDH/toxin EIA results were not reported to wards and not used for patient management. It therefore had to be excluded from the cost comparison study as it would not have impacted on patient LOS.

\section{Length of Hospital Stay}

As main outcome, overall LOS from admission to discharge, LOS from date of stool sample

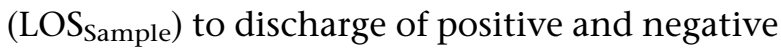
intervention (i.e., PCR) and historic control (i.e., CCNA) samples were compared. LOS data were gathered using the Myrddin Patient Administration System and the in-house Laboratory Information System used routinely at the two hospital sites and recorded anonymously. Data were log-transformed using SPSS 16.0 (IBM Corporation, Armonk, NY, USA) to address skewness of data and differences in duration of inpatient stay between the groups were analyzed using oneway analysis of variance (ANOVA). Average hospital inpatient day costs were obtained from National Health Service (NHS) reference costs (2011) [18] and weighted for specialty and activity.

\section{Cost of Laboratory Testing}

We collected costs in Pound (£) Sterling in 2011 adopting an NHS perspective. Cost of the different tests was estimated using a microcosting bottom-up approach including data 
collection on resource use and costs of materials, capital, waste, repeat samples, overheads, staff time, and staff training time. Resource use for each diagnostic test was obtained by studying the standard operating procedures and observing laboratory staff for a day while they were performing the tasks required for sample processing and evaluation. Unit costs were applied according to information from purchasing records, hospital personnel records and statistics as well as manufacturers and wholesalers. A yearly workload of 10,000 samples was assumed for costing calculations based on laboratory statistics which showed that in 2011, 10,769 samples were tested using CCNA which was the routine method in ABMUHB at that time. A detailed break-down of all collected costs including unit costs, resource use, calculations and assumptions made und source of information can be found in Appendix 1 in the electronic supplementary material (ESM).

\section{Cell Culture Cytotoxicity Neutralization Assay (CCNA)}

In Swansea, until April 2012, CCNA had been the routine test for $C$. difficile in all diarrheal specimens for over 30 years. The stool sample was diluted 1:10 in phosphate buffered saline (PBS), vortexed, and then centrifuged at $3,000 \mathrm{rpm}$ for $20 \mathrm{~min}$. A microtiter plate of Vero cells in 2 ' fetal calf maintenance medium buffered with HEPES (4-(2-hydroxyethyl)-1piperazineethanesulfonic acid) was prepared. Centrifuged PBS extracts of the feces were added to the plate throughout the day using two wells per sample, one of them containing antitoxin neutralizing serum. Positive and negative controls were set up on each plate, incubated in $\mathrm{CO}_{2}$, at $36^{\circ} \mathrm{C}$ overnight, examined under the microscope after $6-8 \mathrm{~h}$ and again the next day (including Saturday) and, if negative, read again after $48 \mathrm{~h}$. On weekends, new samples were not set up but stored until Monday. The presence of $C$. difficile toxin $\mathrm{B}$ was confirmed when at least $50 \%$ of the cells showed cytopathic effects in the test well but not in the neutralized antitoxin well.

\section{Xpert C. difficile PCR Assay}

Stool specimens were directly tested on the closed GeneXpert random access platform, allowing for an autonomous, fully integrated and automated molecular analysis where extraction, amplification, and identification take place successively in the same cartridge. The assay includes reagents for the detection of C. difficile toxin $\mathrm{B}$, binary toxin, and $\mathrm{tcd}$ deletion nt117 as well as the sample processing control. Any Xpert C. difficile assay not yielding a result on the first attempt was repeated using a new cartridge. If no result was obtained upon retesting, the specimen was reported as unresolved and excluded from the study while patient management was decided upon according to clinical diagnosis and the routine CCNA result.

\section{Cost Comparison}

In order to assess potential cost savings or additional costs to the health care service due to the use of real-time PCR for detection of $C$. difficile in stool samples, the number of $C$. difficile samples per year tested in the ABMUHB, number of repeat samples, ratio of positive to negative samples, LOS for the four study groups, and incremental testing costs were considered. A detailed description of all parameters included in the cost calculations can be found in Appendix 2 in the ESM. 
Calculations were set up in Excel $^{\circledR} 2010$ (Microsoft Corporation, Redmond, WA, USA) based on the total number of 10,769 stool samples tested in 2011 (laboratory statistics) in ABMUHB and a rate of positive samples of $2.68 \%$ as 289 positive patients were recorded in 2011 [19]. The assumption was made that initially positive patients were only tested once, while initially negative patients were tested twice if CCNA was used but only once if PCR was used. Uncertainty within the data was addressed by applying the $95 \%$ confidence interval as the range for the LOS results and material costs were reduced by $50 \%$ to account for potential discounts given by manufacturers or wholesalers. Additionally, the total number of samples tested per year was adjusted from 10,000 to 15,000 and 5,000 , respectively, to investigate potential effects of economy of scale on costs. The rate of $C$. difficile-positive patients in ABMUHB in $2011(6.39 / 1,000$ admission $>65$ years) was below the all Wales rate of 7.18 [19]. We therefore changed the percentage of positive samples in our calculations to account for different CDI rates by doubling and halving the percentage of positive tests. We also tested the impact of the assumption that all initially CCNA-negative patients would be retested once by applying the assumption that no retesting was done for any samples and increasing the number of repeat tests to two. This prospective interventional clinical study was approved by the Public Health Wales Research \& Development committee. Ethical approval was not deemed necessary as the specimens were routinely requested according to ABMUHB policy for clinical diagnosis, no additional specimens were collected for study purposes and the commercial diagnostic tests used in the study received CE (Conformité Européenne) marking for the diagnosis of CDI.

\section{RESULTS}

Five-hundred and twenty patients were included in the study of which 14 had to be excluded due to missing LOS data. While we had planned to include the first 150 positive patients, only 121 tested positive in the course of the clinical study. Thus, data of 506 patients were analyzed with 267 in the PCR group and 239 in the CCNA group. There were no significant differences between groups for patient age and gender. Mean age of patients tested by PCR was 75.01 years with $50.6 \%$ male; while mean age of CCNA tested control patients was 74.84 years with $40.7 \%$ male participants. Co-morbidities were similar across the groups. The mean time until results could be reported to the wards was $1.53 \mathrm{~h}$ for PCR, $22.45 \mathrm{~h}$ for positive CCNA, and $46.54 \mathrm{~h}$ for negative CCNA. Average time to results for GDH/toxin EIA was $4.47 \mathrm{~h}$. GDH results were not reported to wards during the study, therefore no LOS data could be linked to these results.

Based on micro-costing, testing cost per sample was $£ 36.18$ for PCR, $£ 7.53$ for CCNApositive, and $£ 8.78$ for CCNA-negative samples (Table 1). Staff hands-on time from sample reception in the laboratory to reporting of the results was more than five times more for CCNA compared to PCR for negative samples and four times more for positive samples (Table 1). During the clinical study, $3.14 \%(33 / 1,051)$ of samples tested by PCR did not yield a result at the first attempt. Of these, 11 had to be excluded from analysis due to insufficient sample and 7 (all mucoid) samples produced errors at second attempt. Cost of these repeat samples was included in the overall PCR costing (see Appendix 1 in the ESM). PCR-positive patients were discharged on average 4.88 days earlier than CCNA-positive patients based on overall LOS and 4.33 days earlier when based on 
Table 1 Costs and resource utilization of PCR and CCNA testing for Clostridium difficile infection per sample (based on 10,000 samples a year)

\begin{tabular}{llll}
\hline Resource & PCR & CCNA & \\
\cline { 3 - 4 } & Positive/negative & Positive & Negative \\
\hline Material cost (including waste and repeat samples) $(£)$ & 34.59 & 2.08 & \\
Capital and overheads $(£)$ & 1.02 & 2.58 & \\
Staff cost (including training) $(£)$ & 0.57 & 2.87 & 4.11 \\
Overall test cost $(£)$ & 36.18 & 7.53 & 8.78 \\
Incremental cost of PCR compared to CCNA per test $(£)$ & $\mathrm{n} / \mathrm{a}$ & 28.65 & 27.40 \\
Total hands-on staff time (sample reception to reporting) $(\mathrm{min})$ & 3.82 & 15.27 & 20.27 \\
Average time to reportable result (sample reception to reporting) $(\mathrm{h})$ & 1.53 & 22.45 & 46.54 \\
\hline
\end{tabular}

CCNA cell culture cytotoxin neutralization assay, $n / a$ not applicable, $P C R$ polymerase chain reaction

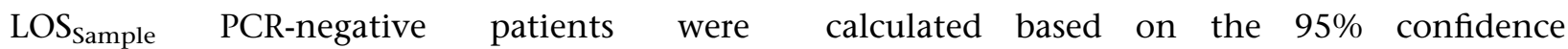
discharged a mean 7.03 days earlier than interval of the LOS results. Cost saving results CCNA-negative patients considering overall LOS and 6.86 days earlier when LOS was calculated from date of sample collection (Table 2). None of these differences were statistically significant ( $P$ values $0.151-0.822$ ). Log-transformation of the skewed LOS data (range 2-340 days) in order to meet the assumption of normality and retesting with ANOVA did not change the results.

Applying the mean values for LOS differences in our calculations (Appendix 2 in the ESM), routine use of real-time PCR had the potential to save 38,247 bed days in ABMUHB in 2011 with the main proportion of this figure (96\%) being contributed by shorter LOS of negative patients. Mean cost savings of up to $£ 2,292.62$ per patient could therefore be achieved by routinely using PCR instead of CCNA (see Appendix 2 in the ESM for description of calculations). In order to investigate the robustness of the results, we recalculated cost savings after changing the values of key parameters (see Table 3). Since the LOS results exhibited large variance and the differences were not significant, potential cost savings or additional investments were were found not to be robust when subject to changes in duration of hospital stay of negative patients. All other parameter changes did not significantly alter the results (Table 3 ). Changes in the quantity of samples processed per year did not have a significant effect on cost savings even though a small potential of economies of scale based on capital investment and staff training costs might be more significant for large laboratories with high sample turnover (Table 3). Due to the lack of statistical significance and large range and variance in LOS data, the results of this study cannot definitely confirm that cost savings will be made by using PCR. However, a clear trend can be observed when results are tested for robustness indicating a high potential for savings (Table 3).

\section{DISCUSSION}

Fast and accurate laboratory results have been suggested to impact patient management and infection control measures [20]. The high sensitivity and specificity of PCR-based assays 
Table 2 Length of hospital stay of inpatients suffering from diarrhea following PCR and CCNA testing for Clostridium difficile

\begin{tabular}{lll}
\hline Parameters & CDI positive & CDI negative \\
\hline$n(\mathrm{CCNA})$ & 115 & 124 \\
$n(\mathrm{PCR})$ & 121 & 146 \\
LOS (CCNA) in days; mean (95\% CI) & $47.67(37.85-57.48)$ & $45.52(37.99-53.05)$ \\
LOS (PCR) in days; mean (95\% CI) & $42.79(35.95-49.63)$ & $38.49(32.05-44.92)$ \\
Mean difference in LOS (PCR vs. CCNA); & $-4.88(-19.39-9.62 ;$ & $-7.03(-20.66-6.60 ;$ \\
mean (95\% CI) & $P=0.822)$ & $P=0.545)$ \\
Number of patients in 2011 in ABMUHB & 289 & 5,240 \\
Inpatient days saved per year & $1,410.32$ & $36,837.20$ \\
\hline
\end{tabular}

$A B M U H B$ Abertawe Bro Morgannwg University Health Board, CCNA cell culture cytotoxin neutralization assay, CDI Clostridium difficile infection, CI confidence interval, LOS length of stay, PCR polymerase chain reaction

for C. difficile detection and the rapid turnaround time present a convincing argument for the routine use of these techniques, but the higher acquisition costs per individual test still let laboratories sometimes hesitate to adopt PCR in their CDI testing routine $[15,16]$. In our study, which considered the impact of the testing assay on duration of inpatient stay, Xpert $C$. difficile realtime PCR was found to produce cost savings in almost all scenarios investigated in comparison to CCNA. Although differences in LOS were not statistically significant in this study, a clear trend is visible towards potentially large cost savings when PCR-based methods are used for C. difficile detection in comparison to CCNA. This trend should be further confirmed by future studies adequately powered to overcome the large variance in LOS data. The mean LOS for patients with suspicion of CDI between 38 and 48 days found in this study is higher compared to LOS reported in other studies. Forster et al. [8] reported a median LOS of 34 days, Vonberg et al. [7] found a median LOS of 27 days, Song et al. [10] 22 days, and Campbell et al. [9] stated a mean duration between 21.0 and 29.3 days for patients suffering from CDI acquired in hospital. However, with the exception of Campbell et al. [9], the mean age of patient populations was considerably younger with 63.2 years [8], 55.9 years [7], and 57.6 years [10], compared to 75 years in our study, which may explain the longer LOS due to potentially higher incidence of co-morbidities.

The cost comparison discussed here only considers the cost of diagnostic tests and the change in duration of hospital stay observed in this study. This approach appears valid considering that cost of additional bed days has been identified as the main cost driver in CDI comprising up to $94 \%$ of the overall costs $[21,22]$. However, it may underestimate potential additional cost savings due to cost reductions in antibiotic treatment and isolation days, as found by other studies [23, 24]. Rapid PCR testing has also been suggested to have the potential for cost savings for detection of methicillin-resistant Staphylococcus aureus [25] and sepsis [26] and to result in cost savings of $\$ 1,037$ per patient in infants with fever and cerebrospinal fluid pleocytosis [27]. To our knowledge, this study is the first to publish an investigation of potential cost savings with a 


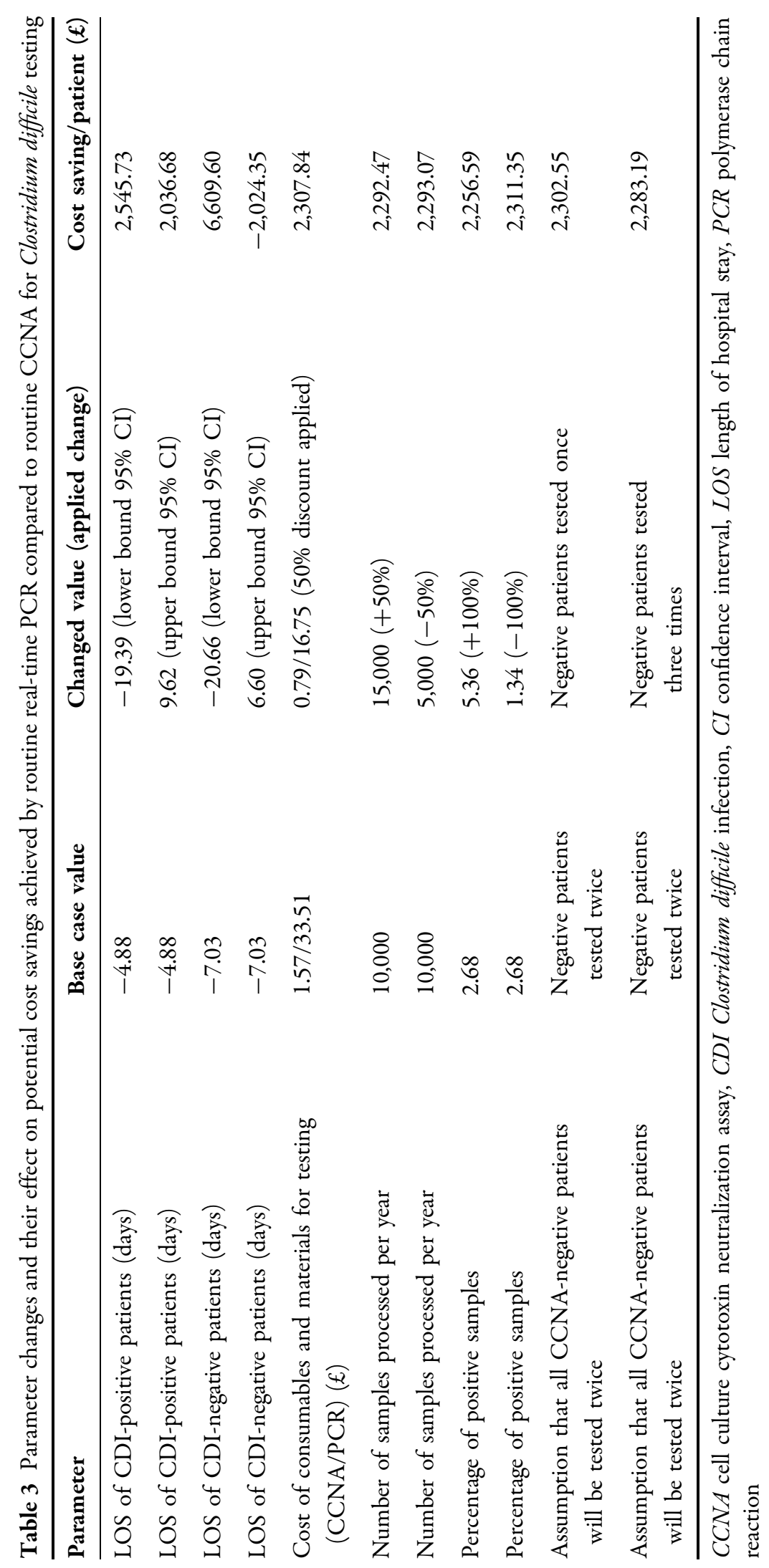


PCR assay for diagnosing CDI compared to CCNA.

The potential cost savings identified in our study may be attributed to the faster turnaround time of PCR-based screening tests allowing for more efficient and accurate patient management, which eventually results in decreased average LOS of 4.88 days for CDI positive and 7.03 for negative patients. Forster et al. [8] suggested that calculating LOS differences based on the overall LOS, not treating $C$. difficile as a time-varying covariable, overestimates the effect of CDI on duration of hospital stay as LOS before CDI will be incorrectly attributed to $C$. difficile. Differences in LOS and $\operatorname{LOS}_{\text {Sample }}$ for patients tested by PCR compared to patients tested by CCNA only differed by less than half a day in our study, suggesting that the decreased LOS for patients tested by PCR can primarily be attributed to the impact of the diagnostic test. GDH/toxin EIA-based assays also have shorter turnaround times and test costs are lower when compared to PCR. However, GDH and toxin EIAs have repeatedly been reported to have a lower sensitivity compared to PCR and CCNA [11, 15, 28-30] despite being widely used and recommended as a two-step algorithm [13, 14]. Our clinical study found that, when compared to clinical diagnosis, $16.2 \%$ of true CDIs were GDH negative and a further $59.7 \%$ of GDH positive, clinically confirmed CDIs were negative in toxin EIA [17]. This is in line with Guerrero et al. [31] and Stahlmann et al. [32] who reported that a third of CDI-positive patients would have been missed using toxin EIA compared to PCR. This is important, as patients with EIA-negative results did not differ in clinical presentation from EIA-positive patients and posed a significant risk for transmission [29]. Considering that around $25 \%$ of CDI patients were suggested to be infected by ward-based patient-to-patient transmission [33, 34], the clinical and financial impact of misidentification of CDI cases would be important. In laboratories using a two-step $\mathrm{GDH} /$ toxin EIA algorithm, costs incurred due to repeat testing performed when the GDH result alone is positive, increased use of antibiotics for those patients with GDH positives which do not confirm with EIA and the increased length of time to a positive toxin result have to be considered. In our clinical study, $35.2 \%$ of patients with GDH-positive specimens did not clinically present CDI [17]. Retesting, treating and isolating patients with false-positive results wastes resources. We observed that GDH failed to pick up a case of CDI, part of a ward outbreak, which was presumptive $C$. difficile ribotype 027 positive with PCR and two GDH-positive 027 cases tested negative by toxin EIA.

The diagnostic accuracy of PCR methods has been established in several trials $[11,15,28,29$, 35]. However, additional positives identified by PCR are often described as false positives when results are only compared to other assays in the laboratory setting and clinical presentation is not considered [36]. Our clinical study showed that out of 59 discrepant samples (CCNA negative but PCR positive), 54 (91.5\%) were found to be true positives on clinical diagnosis which demonstrates convincingly that PCR results are reliable and accurate for diagnosing $\mathrm{CDI}$, at the same time reducing the need for repeat testing. This was confirmed by Napierala et al. [37] who found that after implementation of PCR, testing volume as well as CDI rates decreased significantly. Increased faith of clinicians in a more accurate testing method not only impacts on CDI-positive patients but also affects CDI-negative patients, who can be assessed for other gastrointestinal problems at an earlier point in time without having to revisit $\mathrm{CDI}$ as a cause for diarrhea. Other patients can be 
discharged without further $C$. difficile testing due to the accurate negative results. This was reflected in our study by an average earlier discharge of 7.03 days for PCR-negative patients when compared to matched CCNA control patients. Similar results were reported by Grein et al. [38] who found that average CDI treatment days for negative patients and LOS after CDI diagnosis were shorter with PCR testing compared to toxin EIA and two-step testing.

GDH/toxin EIA results were not reported and thus not used for patient management. Therefore, no direct cost comparison of the GDH followed by toxin EIA algorithm with CCNA and PCR could be performed, which might be considered a limitation of the study. CCNA was used as a reference method as it was the routine test for $C$. difficile detection in the two hospitals at the time of data collection. While it could be criticized that CCNA is not an optimal reference due to its high turnaround time and technical requirements, it has since been shown to correlate well with clinical diagnosis [39]. Our clinical study found a sensitivity and specificity of $99.1 \%$ and $98.9 \%$ for PCR and $51 \%$ and $99.4 \%$ for CCNA, respectively, compared to clinical diagnosis [17]. PCR testing produced 1 false negative and 10 false positives in 1,034 patients compared to CCNA which generated 55 false negatives and 5 false positives. These misidentifications will result in additional resource use and cost due to unnecessary treatment for false positives and repeat testing and increased risk of transmission and spread of infection for false negatives. Whereas repeat testing due to false negative CCNA results was accounted for in the calculations (Appendix 1 in the ESM), additional treatment costs were not considered in this study which could underestimate the cost saving potential of PCR due to the high number of false negatives by
CCNA and the generally higher accuracy of PCR testing [15]. Our study was conducted in two acute hospitals in one trust in Wales and calculations and results are based on figures specific for ABMUHB. While this could limit generalizability of the results, cost savings generated by PCR testing were relatively insensitive to changes in sample quantity, CDI incidence and discount rates on material and consumables required for testing and can therefore be applied to various different laboratory settings in the UK. Even though the sample size of this study was large compared to other studies on CDI, the lack of significance in the LOS differences between the study groups is a major limitation of this study which could be addressed by future studies adequately powered to overcome the large variances in patient LOS observed in our study. Future research should also take into account potential longer term consequences such as CDI recurrences.

\section{CONCLUSION}

The routine use of a rapid molecular test for $C$. difficile in an acute hospital setting produced quick results that led to a decrease in LOS compared to CCNA control patients. While LOS differences were not statistically significant in this study and costs of PCR testing are higher than costs of CCNA per sample, rapid molecular tests can realize potentially large cost savings due to the reduction of excess inpatient days and reduction in cost per total patient care episode.

\section{ACKNOWLEDGMENTS}

The authors wish to thank Dr. Ann Lewis, Stamatela Vagia, Katherine Davies, and Lynne Ray, Microbiology Abertawe Bro Morgannwg 
(Swansea), Delyth Davies, Dr. Salman Jafri, Dr. Chandra Puli, and the ward staff in Singleton and Morriston hospitals, and Dr. Alan Watkins and Prof. Ceri Phillips at Swansea University, and Dr. Anne Postulka for scientific support. The project was supported by a nonpromotional grant for educational purposes from Cepheid Inc., Sunnyvale, CA, USA, and partially funded by Abertawe Bro Morgannwg University Health Board (ABMUHB), Swansea, UK. Cepheid Inc. has provided the funds to cover the publication charges for this article. All named authors meet the ICMJE criteria for authorship for this manuscript, take responsibility for the integrity of the work as a whole, and have given final approval to the version to be published. Parts of this paper were presented as a poster at the 22nd European Congress of Clinical Microbiology and Infectious Diseases (ECCMID) 2012 in London (poster number 2274).

Conflict of interest. Nidhika Berry has received speaker fee and sponsorship for attendance at educational meetings from Cepheid and Astellas. Bernadette Sewell, Eugene Rees, Ian Thomas, Chin Lye Ch'ng, and Mike Isaac declare that they have no conflict of interest.

Compliance with ethics guidelines. All procedures followed were in accordance with the ethical standards of the responsible committee on human experimentation (institutional and national) and with the Helsinki Declaration of 1975 , as revised in 2000 and 2008. This study was approved by the Public Health Wales Research and Development Committee. Ethical approval and informed consent were not deemed necessary as the specimens were requested routinely in accordance with the ABMUHB $C$. difficile care pathway for clinical diagnosis and management, and no additional specimens were collected for study purposes.

Open Access. This article is distributed under the terms of the Creative Commons Attribution Noncommercial License which permits any noncommercial use, distribution, and reproduction in any medium, provided the original author(s) and the source are credited.

\section{REFERENCES}

1. Bartlett JG. Narrative review: the new epidemic of Clostridium difficile-associated enteric disease. Ann Intern Med. 2006;145:758-64.

2. Dubberke ER, Wertheimer AI. Review of current literature on the economic burden of Clostridium difficile infection. Infect Control Hosp Epidemiol. 2009;30:57-66.

3. McGlone SM, Bailey RR, Zimmer SM, et al. The economic burden of Clostridium difficile. Clin Microbiol Infect. 2012;18:282-9.

4. Kyne L, Hamel MB, Polavaram R, Kelly CP. Health care costs and mortality associated with nosocomial diarrhea due to Clostridium difficile. Clin Infect Dis. 2002;34:346-53.

5. Dubberke ER, Reske KA, Olsen MA, McDonald LC, Fraser VJ. Short- and long-term attributable costs of Clostridium difficile-associated disease in nonsurgical inpatients. Clin Infect Dis. 2008;46:497-504.

6. Wilcox MH, Cunniffe JG, Trundle C, Redpath C. Financial burden of hospital-acquired Clostridium difficile infection. J Hosp Infect. 1996;34:23-30.

7. Vonberg RP, Reichardt P, Behnke M, Schwab F, Zindler S, Gastmeier P. Costs of nosocomial Clostridium difficile-associated diarrhoea. J Hosp Infect. 2008;70:15-20.

8. Forster AJ, Taljaard M, Oake N, Wilson K, Roth V, van Walraven $C$. The effect of hospital-acquired infection with Clostridium difficile on length of stay in hospital. CMAJ. 2012;184:37-42.

9. Campbell R, Dean B, Nathanson B, Haidar T, Strauss M, Thomas S. Length of stay and hospital costs among high-risk patients with hospital-origin Clostridium difficile-associated diarrhea. J Med Econ. 2013;16:440-8. 
10. Song X, Bartlett JG, Speck K, Naegeli A, Carroll K, Perl TM. Rising economic impact of Clostridium difficile-associated disease in adult hospitalized patient population. Infect Control Hosp Epidemiol. 2008;29:823-8.

11. Chapin KC, Dickenson RA, Wu F, Andrea SB. Comparison of five assays for detection of Clostridium difficile toxin. J Mol Diagn. 2011;13: 395-400.

12. Planche $T$, Wilcox $M$. Reference assays for Clostridium difficile infection: one or two gold standards? J Clin Pathol. 2011;64:1-5.

13. Cohen SH, Gerding DN, Johnson S, et al. Clinical practice guidelines for Clostridium difficile infection in adults: 2010 update by the Society for Healthcare Epidemiology of America (SHEA) and the Infectious diseases Society of America (IDSA). Infect Control Hosp Epidemiol. 2010;31(5):431-55.

14. Quinn CD, Sefers SE, Babiker W, et al. C. Diff Quik Chek Complete Enzyme Immunoassay Provides a Reliable First-Line Method for detection of Clostridium difficile in Stool Specimens. J Clin Microbiol. 2010;48:603-5.

15. Novak-Weekley SM, Marlowe EM, Miller JM, et al. Clostridium difficile testing in the clinical laboratory by use of multiple testing algorithms. J Clin Microbiol. 2010;48:889-93.

16. Reller M, Alcabasa RC, Lema CA, Carroll KC. Comparison of two rapid assays for Clostridium difficile common antigen and a $\mathrm{C}$ difficile toxin $\mathrm{A} / \mathrm{B}$ assay with the cell culture neutralization assay. Microbiol Infect Dis. 2010;133:107-9.

17. Berry N, Sewell B, Jafri S, Puli C, Vagia S, Lewis AM, Davies D, Rees E, Ch'ng CL. Real-time polymerase chain reaction correlates well with clinical diagnosis of Clostridium difficile infection. J Hosp Infect. 2014;87(2):109-14.

18. Department of Health. NHS reference costs 2010-2011. Department of Health 2012, United Kingdom. http://data.gov.uk/dataset/nhs-referencecosts-2010-11 Accessed Feb 2012.

19. Welsh Healthcare Associated Infection Programme. Clostridium difficile. Mandatory Surveillance Report. Abertawe Bro Morgannwg University Health Board. Public Health Wales. 2012. http://www2.nphs. wales.nhs.uk:8080/WHAIPDocs.nsf/3dc04669c9e1e aa880257062003b246b/87239a685280fb9680257a4 f0035fc37/\$FILE/ABMU\%20Report\%20Apr\%2011\% 20-\%20Mar\%2012.pdf Accessed Jul 2012.

20. Gerding DN, Muto CA, Owens RC. Measures to control and prevent Clostridium difficile infection. Clin Infect Dis. 2008;46:S43-9.
21. Wiegand PN, Nathwani D, Wilcox MH, Stephens J, Shelbaya A, Haider S. Clinical and economic burden of Clostridium difficile infection in Europe: a systematic review of healthcare-facility-acquired infection. J Hosp Infect. 2012;81:1-14.

22. Al-Eidan FA, McElnay JC, Scott MG, Kearney MP. Clostridium difficile-associated diarrhoea in hospitalised patients. J Clin Pharm Ther. 2000;25:101-9.

23. Casari E, Ferrario A, De Luca C, Calabro M, Allibardi $\mathrm{S}$, Lagioia $\mathrm{M}$. Improvement in patient management through the use of a Clostridium difficile PCR real time stand alone test in acute hospital setting. In: Program and Abstracts of the 52nd International Congress of Antimicrobial Agents and Chemotherapy (ICAAC); September 9-12, 2012; San Francisco, CA. Abstract D-159.

24. Bartsch SM, Curry SR, Harrison LH, Lee BY. The potential economic value of screening hospital admissions for Clostridium difficile. Eur J Clin Microbiol Infect Dis. 2012;31:3163-71.

25. Brown J, Paladino JA. Impact of rapid methicillinresistant Staphylococcus aureus polymerase chain reaction testing on mortality and cost effectiveness in hospitalised patients with bacteraemia. Pharmacoeconomics. 2010;28:567-75.

26. Lehmann LE, Herpichboehm B, Kost GJ, Koller MH, Stueber F. Cost and mortality prediction using polymerase chain reaction pathogen detection in sepsis: evidence from three observational trials. Crit Care. 2010;14:R186.

27. Nigrovic LE, Chiang VW. Cost analysis of enteroviral polymerase chain reaction in infants with fever and cerebrospinal fluid pleocytosis. Arch Pediatr Adolesc Med. 2000;154:817-21.

28. Eastwood K, Else P, Charlett A, Wilcox M. Comparison of nine commercially available Clostridium difficile toxin detection assays, a realtime PCR assay for C. difficile tcdB and a glutamate dehydrogenase detection assay to cytotoxin testing and cytotoxigenic culture methods. J Clin Microbiol. 2009;47:3211-7.

29. Sloan LM, Duresko BJ, Gustafson DR, Rosenblatt JE. Comparison of real-time PCR for detection of the tcdC gene with four toxin immunoassays and culture in diagnosis of Clostridium difficile infection. J Clin Microbiol. 2008;46:1996-2001.

30. Humphries RM, Uslan DZ, Rubin Z. Performance of Clostridium difficile toxin enzyme immunoassay and nucleic acid amplification tests stratified by patient disease severity. J Clin Microbiol. 2013;51(3):869-73.

31. Guerrero DM, Chou C, Jury LA, Nerandzic MM, Cadnum JC, Donsky CJ. Clinical and infection 
control implications of Clostridium difficile infection with negative enzyme immunoassay for toxin. Clin Infect Dis. 2011;53:287-90.

32. Stahlmann J, Schoenberg M, Herrmann M, von Mueller L. Detection of nosocomial Clostridium difficile infections with toxigenic strains despite negative toxin A and B testing on stool samples. Clin Microbiol Infect. 2014; Jan 23. doi: 10.1111/ 1469-0691.12558.

33. Walker AS, Eyre DW, Wyllie DH, et al. Characterisation of Clostridium difficile hospital ward-based transmission using extensive epidemiological data and molecular typing. PLoS Med. 2012;9:e1001172.

34. Lanzas C, Dubberke ER, Lu Z, Reske KA, Gröhn YT. Epidemiological model for Clostridium difficile transmission in healthcare settings. Infect Contr Hosp Epidemiol. 2011;32:553-61.

35. Huang $H$, Weintraub $A$, Fang $H$, Nord CE. Comparison of a commercial multiplex real-time PCR to the cell cytotoxicity neutralization assay for diagnosis of Clostridium difficile infections. J Clin Microbiol. 2009;47:3729-31.

36. Buchan BW, Mackey T-LA, Daly JA, et al. Multicenter clinical evaluation of the Portrait toxigenic C. difficile assay for detection of toxigenic Clostridium difficile in clinical stool specimens. J Clin Microbiol. 2012;50:3932-6.

37. Napierala M, Munson E, Skonieczny P, et al. Impact of toxigenic Clostridium difficile polymerase chain reaction testing on the clinical microbiology laboratory and inpatient epidemiology. Diagn Microbiol Infect Dis. 2013;76:534-8.

38. Grein JD, Ochner M, Hoang H, Jin A, Morgan MA, Murthy AR. Comparison of testing approaches for Clostridium difficile infection at a large community hospital. Clin Microbiol Infect. 2014;20:65-9.

39. Planche TD, Davies KA, Coen PG, et al. Differences in outcome according to Clostridium difficile testing method: a prospective multicentre diagnostic validation study of $\mathrm{C}$ difficile infection. Lancet Infect Dis. 2013;13:936-45. 